\title{
Traction Power Generation with Tethered Wings
}

Michael Noom, Roland Schmehl, Rolf van der Vlugt

Delft University of Technology, Faculty of Aerospace Engineering

A tethered wing can be used in two different ways, to lift payload or to provide traction power. The latter is the basis of several innovative technical applications, such as kite-assisted ship propulsion and pumping-kite wind energy conversion. We present a theoretical analysis of traction power generation by a tethered wing. The objective is to establish the fundamental relationships between system and operational parameters on the one hand, and the achievable mechanical power output on the other hand. It is assumed that the instantaneous flight state of the wing can be approximated by the steady equilibrium of aerodynamic and tether forces.
The analysis covers the general case of controlled flight along an arbitrary, predefined trajectory with varying tether length. The analysis is compared with several special cases that are available in literature. In a second step, the effect of weight and centrifugal acceleration of the wing is included in the analysis, as well as the effect of weight and aerodynamic drag of the tether. To estimate the potential traction power generation the analysis is applied to the specific trajectory of a pumping cycle, which is characterized by a traction phase, in which the kite performs figureeight maneuvers, a retraction phase and a transition phase.

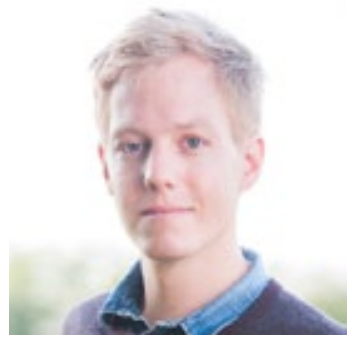

Michael Noom

Delft University of Technology Faculty of Aerospace Engineering

Wind Energy Section

Kite Power Research Group

Kluyverweg 1

2629 HS Delft

The Netherlands

michael.noom@gmail.com

http://www.kitepower.eu

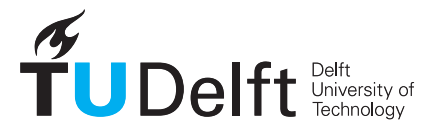

KitePower 